\title{
Nach erstem Schlaganfall reicht ASS nicht mehr
}

\author{
Wenn es unter Primärprophylaxe mit ASS zu einem Schlaganfall kommt, sollte die \\ antithrombotische Therapie auf Clopidogrel mit oder ohne ASS umgestellt werden.
}

_ Viele Patienten nehmen zur Primärprophylaxe vaskulärer Komplikationen Acetylsalizylsäure (ASS) ein. Für eine südkoreanische Studie wurden die Daten von 1.172 Patienten im mittleren Alter von 69,7 Jahre analysiert, die unter einer präventiven ASS-Einnahme einen nicht-embolischen Schlaganfall erlitten. Keiner von ihnen wurde während oder nach dem Krankenhausaufenthalt antikoaguliert. Nach dem Schlaganfall wurde die antithrombotische Therapie weitergeführt, wobei 212 Patienten weiterhin nur ASS nahmen, 246 zu einer Monotherapie mit Clopidogrel wechselten und 714 mit einer Kombination der beiden Wirkstoffe behandelt wurden.
Der primäre Endpunkt setzte sich aus ischämischen und hämorrhagischen Schlaganfällen, Myokardinfarkten und Todesfällen mit vaskulärer Ursache zusammen und trat innerhalb eines Jahres nach dem ersten Schlaganfall 86-mal ein. Unter Fortführung der ASS-Gabe lag der Anteil bei 14,5\%, in der ClopidogrelGruppe bei 7,4\% und in der Kombinationsgruppe bei $6,7 \%$, also hoch signifikant seltener $(\mathrm{p}=0,001)$. Auch ein erneuter Schlaganfall trat in der ASSGruppe signifikant häufiger auf $(9,0 \%$ vs. $7,4 \%$ vs. $5,0 \%, \mathrm{p}=0,031$ )

- Kim JT, Park MS, Choi KH et al. Different Antiplatelet Strategies in Patients With New Ischemic Stroke While Taking Aspirin. Stroke. 2016;47:128-34

\section{KOMMENTAR}

ASS ist Mittel der ersten Wahl für die Primärprävention kardialer, zerebraler und vaskulärer Komplikationen bei multiplen kardiovaskulären Risikofaktoren. Nach den aktuellen Leitlinien der American Heart Association und der American Stroke Association gab es bisher keine Hinweise, wie die antithrombotische Therapie nach einem ersten Schlaganfall unter ASS am besten weitergeführt wird. Die vorliegende Studie aus Korea an einer begrenzten Patientenzahl favorisiert für die Sekundärprophylaxe den Wechsel von ASS zur Monotherapie mit Clopidogrel oder zur Kombination von ASS plus Clopidogrel. Zur Häufigkeit von Blutungen fehlen Angaben.

Prof. Dr. med. H. Holzgreve

\section{Seltene Dislokation nach einem Sturz}

Eine 48-jährige Frau war ausgerutscht und auf die rechte Hand gefallen. Sie klagte über Schmerzen an der Unterseite des rechten Handgelenks, die sich bei Bewegung verstärkten. Bei
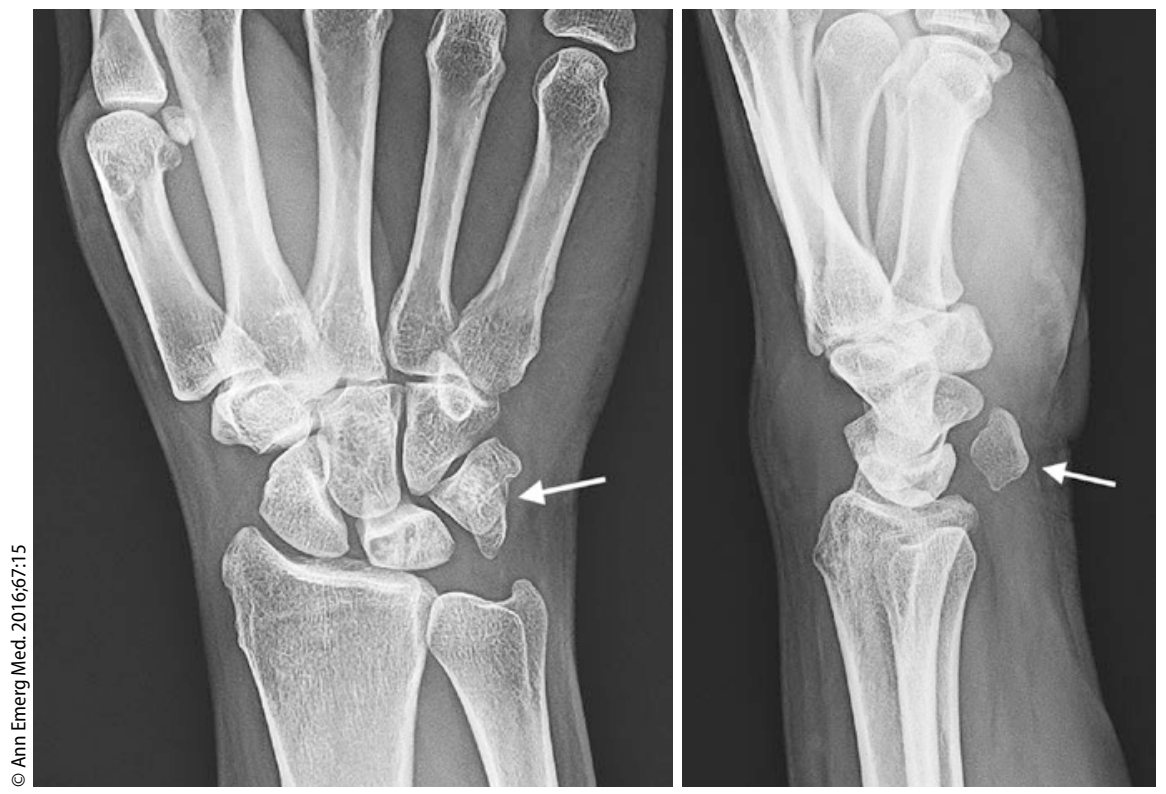

Disloziertes Os pisiforme (Pfeile) im Röntgenbild der Hand. im Bereich der Handfläche und ulnarseitig am Handgelenk sowie einen umschriebenen Druckschmerz im Bereich des Hypothenar am der Untersuchung sah man ein leichtes Ödem

distalen Ulnaende. Die Ulnardeviation und -flexion waren besonders schmerzhaft. Pulsstatus und Trophik waren unauffällig. Auf den Bildern in zwei Ebenen des rechten Handgelenks erkannte man eine Dislokation des Os pisiforme.

Die isolierte Dislokation des Os pisiforme ist sehr selten. Das Os pisiforme ist ein Sesambein, das mit dem Os triquetrum flach artikuliert, seine Stabilität aber überwiegend durch die Sehne des $M$. flexor carpi ulnaris erhält, die sich nach distal in das Ligamentum pisohamatum und pisometacarpale fortsetzt. Eine Dislokation wird entweder über direkte Gewalteinwirkung auf das Os pisiforme selbst bewirkt, was üblicherweise zu einer Fraktur desselben führt, oder indirekt durch eine Traktion der Sehne des M. flexor carpi ulnaris. Behandlungsoptionen sind u. a. die Ruhigstellung in einem Splint mit oder ohne vorherige manuelle Reposition oder die vollständige Entfernung des Os pisiforme. Die Patientin wurde zunächst nur mit einer Bandage versorgt und erschien nicht mehr zu einer Nachkontrolle.

Prof. Dr. med. H. S. FüeßI

- Baum AE et al. Images in emergency medicine.

Isolated pisiform dislocation. Ann Emerg Med. 2016;67:15 